Comfort Ohajunwa, Kirthi Kumar, and Padmanabhan Seshaiyer*

\title{
Mathematical modeling, analysis, and simulation of the COVID-19 pandemic with explicit and implicit behavioral changes
}

https://doi.org/10.1515/cmb-2020-0113

Received September 5, 2020; accepted December 21, 2020

Abstract: As COVID-19 cases continue to rise globally, many researchers have developed mathematical models to help capture the dynamics of the spread of COVID-19. Specifically, the compartmental SEIR model and its variations have been widely employed. These models differ in the type of compartments included, nature of the transmission rates, seasonality, and several other factors. Yet, while the spread of COVID-19 is largely attributed to a wide range of social behaviors in the population, several of these SEIR models do not account for such behaviors. In this project, we consider novel SEIR-based models that incorporate various behaviors. We created a baseline model and explored incorporating both explicit and implicit behavioral changes. Furthermore, using the Next Generation Matrix method, we derive a basic reproduction number, which indicates the estimated number of secondary cases by a single infected individual. Numerical simulations for the various models we made were performed and user-friendly graphical user interfaces were created. In the future, we plan to expand our project to account for the use of face masks, age-based behaviors and transmission rates, and mixing patterns.

Keywords: COVID-19, Compartmental Model, Social Behavior

MSC: 92-08, 92BXX, 34AXX

\section{Introduction}

COVID-19 has been a pandemic of monumental proportions. Ever since the identification of the cause of the outbreak of COVID-19 in late 2019 and its pandemic designation in March of 2020 [14, 12], research and development activities have been evolving into a broader understanding of the epidemiology of the novel coronavirus as a "super-spreader" of infectious disease. Specifically, there have been several mathematical models that have been proposed to forecast the spread as well as the future of the coronavirus disease 2019 (COVID19) epidemics in the US and worldwide. The primary and most effective use of these epidemiological models have been to estimate the relative effect of various interventions in reducing disease burden rather than to produce precise quantitative predictions about extent or duration of disease burdens.

Mathematical models have been used by several researchers to study the transmission dynamics of infectious diseases. A typical framework for many of the mathematical models include employing classical compartmental models involving an ordinary differential equation (ODE) system using a Susceptible-ExposedInfected-Recovered (SEIR) compartmental structure for modeling the spread of a disease [1]. Ever since the news of COVID-19 was traced back to Wuhan, several researchers tried to model the dynamics using phenomenological models [13] and variations of the SEIR model [6] to analyze this epidemic. Many of the papers have studied public health interventions within the SIR/SEIR such as focusing on social distancing [11]. Most

Comfort Ohajunwa: George Mason University, Fairfax, Virginia, USA

Kirthi Kumar: University of California, Berkeley, California, USA

*Corresponding Author: Padmanabhan Seshaiyer: George Mason University, Fairfax, Virginia, USA,

E-mail: pseshaiy@gmu.edu 
of these models make many key assumptions based on limited data. While they may have captured aspects of epidemics effectively they often neglected to account for other factors, such as the effect of undetected infected people [8]; effect of containment strategies [10]; details of immunity to disease; reinfection potential; or population characteristics, such as age distribution, population with co-morbidities, and risk factors (e.g., smoking, exposure to air pollution). Some critical variables, including the reproductive number (the average number of new infections associated with one infected person) and social distancing effects, can also change over time. Most importantly, many of these models do not capture the effect of social behavior and how that impacts the spread of the disease, which is the focus of this work.

It is important to realize that COVID-19 spread can impact behavior in the population. For example, people could decide to confine at home as a government lockdown continues, wash their hands more frequently, wear protective masks, and avoid crowded places and schools, theaters, bars, and libraries could be closed, social and sports events could be canceled, people could stay home from work, and businesses could close. Surprisingly, most of the mathematical models do not explicitly include the occurrence of these behavior changes. Without including behavioral changes, the simulations will predict the 'worst' possible scenario. The impact of individual and community behavioral changes in response to an outbreak of a disease like COVID-19 with high mortality needs to be therefore taken into account while modeling.

The main goal of this paper is to develop a new mathematical model well adapted to COVID-19 taking into account the the impact of social behavior. This model will expand on earlier models that have been established for coronavirus transmission dynamics [7, 9]. The model developed herein, is complex enough to capture most dynamic interactions, but also simple enough to allow an affordable identification of its parameters from available data that can help build policy in response to the spread of this pandemic.

The outline of the paper is as follows. In Section 2, we present the mathematical framework used to study the transmission dynamics of COVID-19. Here we present a baseline model that introduces the main states of the human sub-population through an enhanced SEIR model for COVID-19. For this model, the basic reproduction number is derived using a Next Generation matrix approach. In section 3, the baseline model is then enhanced using an explicit intervention model with social behavior under lockdown conditions. Section 4 presents an implicit intervention model again expanding on the baseline model, that incorporates social behaviour into the transmission parameter. Section 5 presents numerical experiments for the various models presented in the paper. We also develop Graphical User Interfaces that can serve as interactive dashboards for simulating all models developed in this work for public use.

\section{Mathematical Model and Governing Equations}

\subsection{A Baseline COVID-19 Model}

In this work, an extended SEIR mathematical model is presented that incorporates multiple classes of infections in humans, asymptomatic and symptomatic, which are assumed to be equally infectious and of similar duration (period of infectiousness) as well as quarantine, recovered, hospitalized and dead sub-populations. We also keep track of the number of deaths through a separate compartment. For simplicity, the model neglects natural birth and death rates. The baseline model is organized around the following flow diagram (see Figure 1). The model assumes the following human sub-populations that include:

- Susceptible (S): Individuals who have not been infected with COVID-19 and have not isolated themselves from the population

- Exposed $(E)$ : Individuals in the incubation period of disease progression

- Asymptomatic $\left(I^{A}\right)$ : Infectious individuals who do not show symptoms of COVID-19

- Symptomatic $\left(I^{S}\right)$ : Infectious individuals who show symptoms of COVID-19

- Hospitalized $(H)$ : Infectious individuals, hospitalized with symptoms of COVID-19

- Quarantine $(Q)$ : Symptomatic infectious individuals who are isolated

- Recovered (R): Individuals who survived COVID-19 


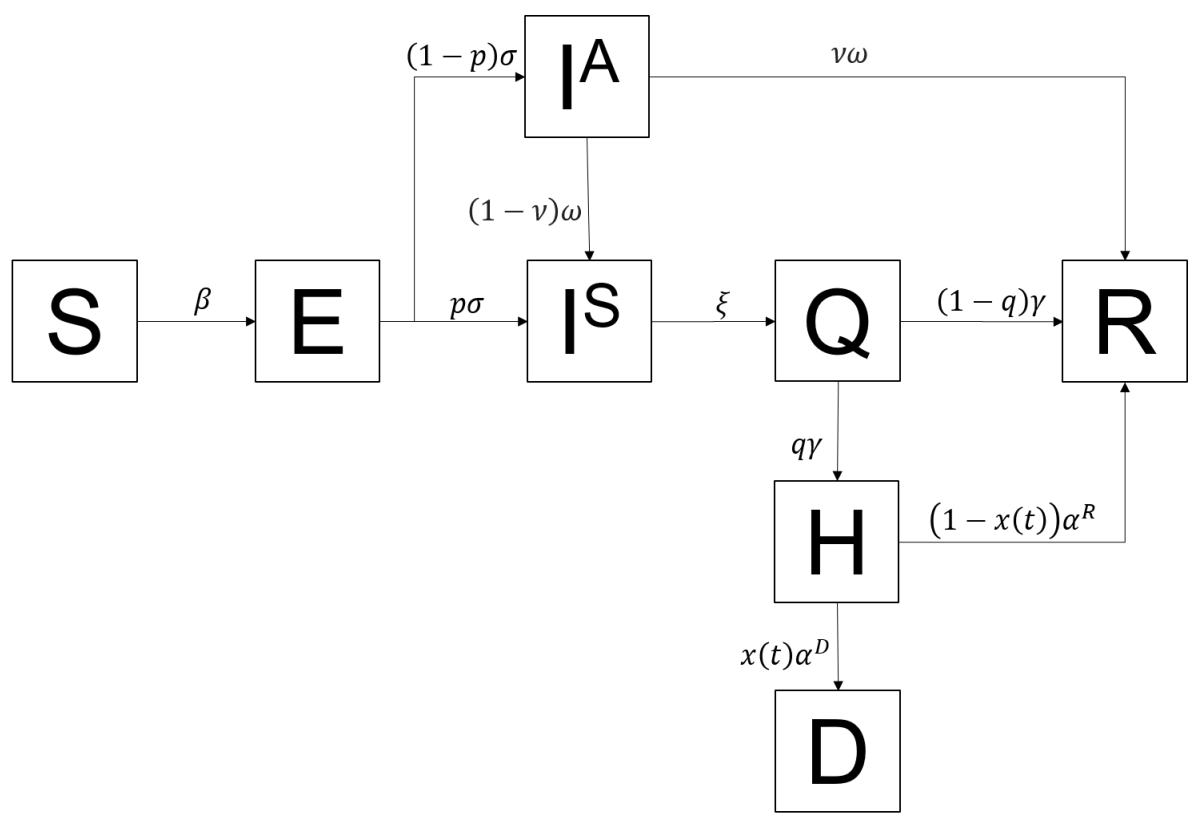

Figure 1: Flow diagram of a Baseline COVID-19 Model

- Dead (D): Individuals who did not survive COVID-19

We will assume that the states $H$ and $Q$ no longer spread COVID-19. This model is described with the following governing equations:

$$
\begin{aligned}
\frac{d S}{d t} & =-\beta S\left(\frac{I^{A}+I^{S}}{N}\right) \\
\frac{d E}{d t} & =\beta S\left(\frac{I^{A}+I^{S}}{N}\right)-\sigma E \\
\frac{d I^{A}}{d t} & =(1-p) \sigma E-\omega I^{A} \\
\frac{d I^{S}}{d t} & =p \sigma E+(1-v) \omega I^{A}-\xi I^{S} \\
\frac{d H}{d t} & =q \gamma Q-\left((1-x) \alpha^{R}+x \alpha^{D}\right) H \\
\frac{d Q}{d t} & =\xi I^{S}-\gamma Q \\
\frac{d R}{d t} & =v \omega I^{A}+(1-q) \gamma Q+(1-x) \alpha^{R} H \\
\frac{d D}{d t} & =x \alpha^{D} H
\end{aligned}
$$

In a population of $N=S+E+I^{A}+I^{S}+H+Q+R+D$ humans, susceptible individuals $S$ move to the exposed class $E$ after acquiring the COVID-19 disease through interaction with an infected individual. This transmission is being modeled via the addition of terms directly proportional to the respective infected human classes (asymptomatic $I^{A}$ and symptomatic $I^{S}$ ) involved in the transmission and an infection rate proportional to the infected individuals. The rate of transmission from the infected to susceptible humans is given by the usual product $\beta$. Note that the exposed category models the incubation period before a human becomes infectious, contrasting the asymptomatic $I^{A}$ and symptomatic $I^{S}$ categories. Members of the exposed class $E$ move to become either symptomatic infectious or asymptomatic infectious at a human incubation rate proportional to $\sigma$ which is the intrinsic human latent period. A fraction $(1-p)$ of the latent becomes 
asymptomatic infectious and a fraction $p$ become symptomatic infectious. Members of the asymptomatic $I^{A}$ class recover with a rate proportional to $\omega$ with a fraction $(1-v)$ moving to become symptomatic $I^{S}$ infectious and the fraction $v$ moving to the recovered class. Symptomatic individuals become quarantined at a rate of $\xi$. Individuals leave the quarantine compartment at a rate proportional to $\gamma$ with a fraction $q$ becoming hospitalized and a fraction $(1-q)$ becoming recovered. Finally, hospitalized individuals die at a rate of $x$ and are hospitalized for a duration of $1 / \alpha^{D}$ while hospitalized individuals recover at a rate of $(1-x)$ and are hospitalized for a duration of $1 / \alpha^{R}$. The death rate, $x=x(t)$, is defined as a function of time:

$$
x(t)= \begin{cases}\hat{x} & \text { if } H \leq B, \\ \frac{\hat{x} B+H-B}{H} & \text { if } H>B,\end{cases}
$$

where $B$ is the number of ICU beds and $\hat{x}$ is the lethality rate of individuals who have access to an ICU bed. Note that the first part of the above function represents when the number of hospitalized individuals is less than or equal to the number of beds. In other words, there is enough beds for all hospitalized individuals. The second part of the function represents when there is a shortage of beds such that the number of hospitalized individuals is greater than the number of beds. The definition of the parameters are given in Table 1.

Table 1: Definition of Parameters in the Baseline COVID-19 model

\begin{tabular}{cl}
\hline Parameter & Definition \\
\hline$\beta$ & Transmission Rate \\
$\sigma$ & Rate at which Exposed individuals become Infected \\
$\omega$ & Rate at which Asymptomatic individuals become Symptomatic or Recovered \\
$\xi$ & Rate at which Symptomatic individuals become Quarantined \\
$\gamma$ & Rate at which Quarantined individuals become Hospitalized or Recovered \\
$1 / \alpha^{R}$ & Duration at which Hospitalized individuals who recover remain hospitalized \\
$1 / \alpha^{D}$ & Duration at which Hospitalized individuals who die remain hospitalized \\
$x$ & Rate at which Hospitalized individuals die \\
$\hat{x}$ & Lethality Rate of Hospitalized individuals with access to an ICU Bed \\
$p$ & Fraction of Symptomatic individuals out of Exposed individuals \\
$q$ & Fraction of Hospitalized individuals out of Quarantined individuals \\
$v$ & Fraction of Recovered individuals out of Asymptomatic individuals \\
\hline
\end{tabular}

\subsection{Derivation of the Basic Reproduction Number $\boldsymbol{R}_{0}$}

In this section, we will derive a basic reproduction number $\mathcal{R}_{0}$ that can be used to measure the transmission potential of COVID-19 as proposed by the system (1)-(8). $\mathcal{R}_{0}$ is the average number of secondary infections produced by a typical case of an infection in a population where everyone is susceptible [4].

Let us recall that the proposed mathematical model for COVID-19 includes sub-populations with different infectious states. Therefore, we will employ a general approach called the Next Generation Matrix [1] to find the basic reproduction number $\mathcal{R}_{0}$ which is given by the following theorem.

Theorem 2.1. The basic reproduction number $\mathcal{R}_{0}$ is given by

$$
\mathcal{R}_{0}=R_{0}^{1}+R_{0}^{2}+R_{0}^{3}
$$


where,

$$
\begin{aligned}
& R_{0}^{1}=\beta \cdot \frac{p}{\xi} \\
& R_{0}^{2}=\beta \cdot \frac{(1-p)}{\omega} \\
& R_{0}^{3}=\beta \cdot \frac{(1-v)(1-p)}{\xi}
\end{aligned}
$$

Proof. Given that the infectious states: $E, I^{A}, I^{S}$ in equations (1)-(8), we can create a vector $\mathcal{F}$ that represents the new infections flowing only into the exposed compartments given by:

$$
\mathcal{F}=\left\{\beta\left(I^{A}+I^{S}\right), 0,0\right\}
$$

Along with $\mathcal{F}$, we will also consider $\mathcal{V}$ which denotes the outflow from the infectious compartments in equations (1)-(8) which is given by:

$$
\mathcal{V}=\left\{\sigma E, \omega I^{A}-(1-p) \sigma E, \xi I^{S}-p \sigma E-(1-v) \omega I^{A}\right\}
$$

Next, we compute the Jacobian $F$ from $\mathcal{F}$ given by,

$$
F=\left(\begin{array}{ccc}
0 & \beta & \beta \\
0 & 0 & 0 \\
0 & 0 & 0
\end{array}\right)
$$

and the Jacobian $V$ from $\mathcal{V}$ given by,

$$
V=\left(\begin{array}{ccc}
\sigma & 0 & 0 \\
-(1-p) \sigma & \omega & 0 \\
-p \sigma & -(1-v) \omega & \xi
\end{array}\right)
$$

We can then compute the inverse of the matrix $V$ to be:

$$
V^{-1}=\left(\begin{array}{ccc}
\frac{1}{\sigma} & 0 & 0 \\
\frac{1-p}{\omega} & \frac{1}{\omega} & 0 \\
\frac{(1-v)(1-p)}{\xi}+\frac{p}{\xi} & \frac{1-v}{\xi} & \frac{1}{\xi}
\end{array}\right)
$$

Using matrices $F$ and $V$ one can then compute the Next Generation Matrix $F V^{-1}$ given by:

$$
F V^{-1}=\beta\left(\begin{array}{ccc}
\frac{p}{\xi}+\frac{1-p}{\omega}+\frac{(1-v)(1-p)}{\xi} & \frac{1}{\omega}+\frac{1-v}{\xi} & \frac{1}{\xi} \\
0 & 0 & 0 \\
0 & 0 & 0
\end{array}\right)
$$

Note that $(i, j)$ entry of the Next Generation Matrix $F V^{-1}$ is the expected number of secondary infections in compartment $i$ produced by individuals initially in compartment $j$ assuming that the environment seen by the individual remains homogeneous for the duration of its infection. Also, matrix $F V^{-1}$ is nonnegative and therefore has a non-negative eigenvalue. The basic reproduction number can then be computed as $\mathcal{R}_{0}=\rho\left(F V^{-1}\right)$ which is the spectral radius of the matrix. This non-negative eigenvalue is associated with a non-negative eigenvector which represents the distribution of infected individuals that produce the greatest number $\mathcal{R}_{0}$ of secondary infections per generation.

The basic reproduction number $\mathcal{R}_{0}$ corresponds to the dominant eigenvalue and therefore:

$$
\mathcal{R}_{0}=\beta\left[\frac{p}{\xi}+\frac{1-p}{\omega}+\frac{(1-v)(1-p)}{\xi}\right]
$$


which can be rewritten as the sum of three expressions:

$$
\mathcal{R}_{0}=R_{0}^{1}+R_{0}^{2}+R_{0}^{3}
$$

where,

$$
\begin{aligned}
& R_{0}^{1}=\beta \cdot \frac{p}{\xi} \\
& R_{0}^{2}=\beta \cdot \frac{(1-p)}{\omega} \\
& R_{0}^{3}=\beta \cdot \frac{(1-v)(1-p)}{\xi}
\end{aligned}
$$

Note that Theorem 2.1 yields a general result for the basic reproduction number $\mathcal{R}_{0}$ corresponding to the COVID-19 disease transmission model given by equations (1)-(8). In addition, note that the three expressions $R_{0}^{1}, R_{0}^{2}, R_{0}^{3}$ are written as the product of the transmission rate, the probability of entering an infectious state (symptomatic, asymptomatic, or asymptomatic to symptomatic), and the mean duration of that infectious state, respectively. Thus, each expression represents the average number of secondary cases produced by an infectious individual in a certain infectious state during their infectious period.

Corollary 2.2. Suppose we assume $p=1$. In this case, the basic reproduction number is given by

$$
\mathcal{R}_{0}=\frac{\beta}{\xi}
$$

This assumes all infectious cases are symptomatic. Hence, this basic reproduction number shows the ratio of the transmission rate to the quarantine rate.

\section{An Explicit COVID-19 Model}

Over the past few months, many parts of the globe have seen curfews, quarantines, and similar restrictions (described as stay-at-home orders, shelter-in-place orders, shutdowns, or lockdowns). For modeling lockdown, there have been attempts to consider vertical confinement as an exit strategy to the regular lockdown. In this section we study the impact of introducing a confinement compartment explicitly into the system of equations (1) - (8). This confined group $(C)$ will consist of individuals who have not been infected with COVID19 and have isolated themselves from the population. Specifically, we will assume that lockdown removes a fraction of the susceptible $(S)$ population at a rate given by $\phi=\phi(t)$, making them confined $(C)$.

Figure 2 illustrates the flow diagram of the expansion of the baseline model to the explicit model whose equations may be described by: 


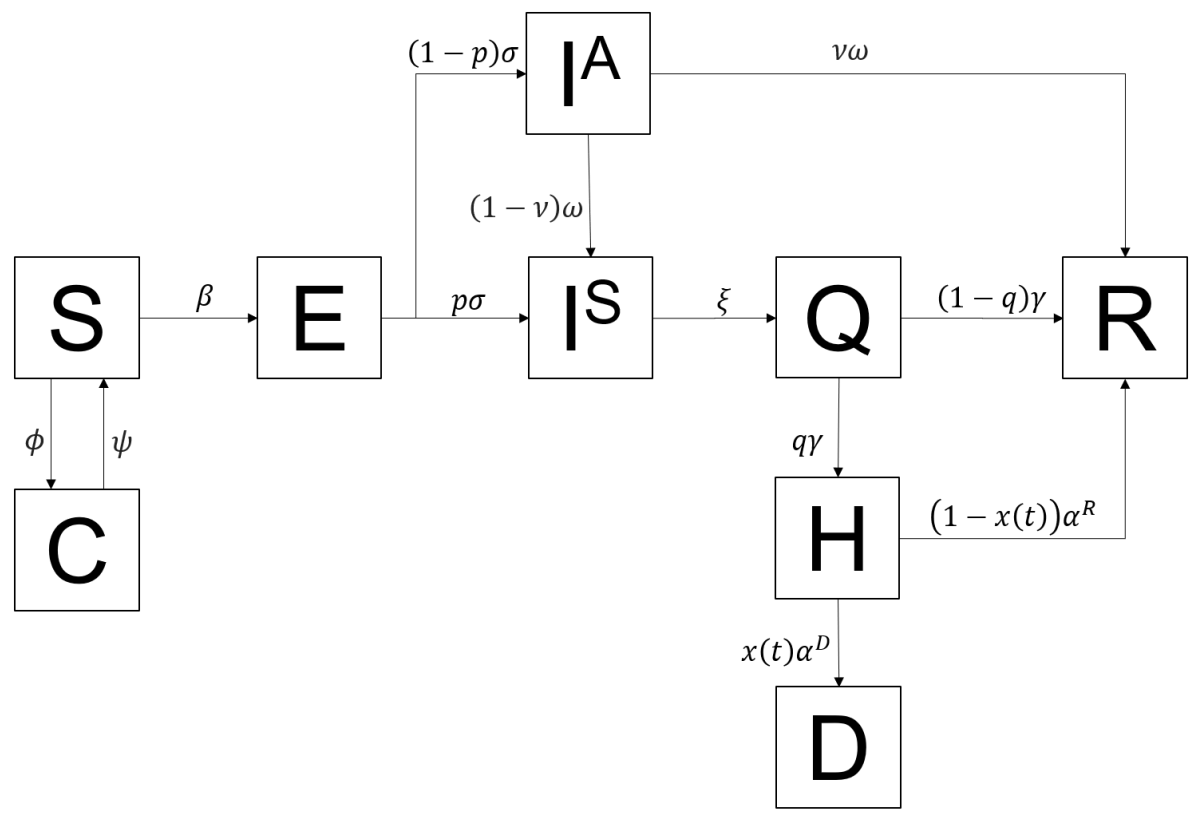

Figure 2: Flow Diagram of an Explicit COVID-19 Model

$$
\begin{aligned}
\frac{d S}{d t} & =-\phi S+\psi C-\beta S\left(\frac{I^{A}+I^{S}}{N}\right) \\
\frac{d C}{d t} & =\phi S-\psi C \\
\frac{d E}{d t} & =\beta S\left(\frac{I^{A}+I^{S}}{N}\right)-\sigma E \\
\frac{d I^{A}}{d t} & =(1-p) \sigma E-\omega I^{A} \\
\frac{d I^{S}}{d t} & =p \sigma E+(1-v) \omega I^{A}-\xi I^{S} \\
\frac{d H}{d t} & =q \gamma Q-\left((1-x) \alpha^{R}+x \alpha^{D}\right) H \\
\frac{d Q}{d t} & =\xi I^{S}-\gamma Q \\
\frac{d R}{d t} & =v \omega I^{A}+(1-q) \gamma Q+(1-x) \alpha^{R} H \\
\frac{d D}{d t} & =x \alpha^{D} H
\end{aligned}
$$

We have assumed susceptible individuals become confined at a rate of $\phi$ and confined individuals become susceptible at a rate of $\psi$. Here $\phi$ and $\psi$ are defined as Dirac Delta functions based on lockdown times:

$$
\begin{gathered}
\phi(t)=a \delta(t-t l o c k) \\
\psi(t)=b \delta(t-t l i f t)
\end{gathered}
$$

where $a$ is the fraction of susceptible individuals that enter confinement, $b$ is the fraction of confined individuals that become susceptible, tlock is the lockdown time in days, and tlift is the lockdown lift time in days. 


\section{An Implicit COVID-19 Model}

In this section, we introduce a new implicit model, in which a population of individuals are subject to COVID19 outbreak developing according to system (1)-(8), but in which contact rates depend on the behavioral patterns adopted across the population. For this new model, we allow susceptible individuals to adopt one of two behaviors: normal behavior or altered behavior. Susceptible individuals with normal behavior $\left(S_{n}\right)$ do not reduce their social contacts, whereas susceptible individuals with altered behavior $\left(S_{a}\right)$ reduce their contacts through interventions such as social distancing and self-isolation. Both groups are assumed to have different transmission rates associated with their behavior: $\beta_{n}$ and $\beta_{a}$, where $\beta_{a}=r \beta_{n}$, with $r$ representing the reduction of contagious contacts associated with altered behavior. We can then model the susceptible individuals adopting behavior $\beta_{n}$ and $\beta_{a}$, respectively as

$$
\begin{aligned}
& \dot{S_{n}}=-\beta_{n} S_{n}\left(\frac{I^{A}+I^{S}}{N}\right) \\
& \dot{S_{a}}=-\beta_{a} S_{a}\left(\frac{I^{A}+I^{S}}{N}\right)
\end{aligned}
$$

Letting the total susceptible population to be $S=S_{a}+S_{n}$, we can rewrite $\dot{S}=\dot{S}_{n}+\dot{S}_{a}$.

Introducing the variable $y$ to denote the proportion of Susceptible individuals adopting normal behavior, given by $y=\frac{S_{n}}{S_{n}+S_{a}}$, we have,

$$
\begin{aligned}
\dot{S} & =\dot{S_{n}+\dot{S}_{a}} \\
& =-\left(\beta_{n} S_{n}+\beta_{a} S_{a}\right)\left(\frac{I^{A}+I^{S}}{N}\right) \\
& =-\beta(y) S\left(\frac{I^{A}+I^{S}}{N}\right)
\end{aligned}
$$

where, $\beta(y)=\beta_{n}[y+r(1-y)]$. Note that $y$ changes according to a natural selection process that benefits susceptible individuals with altered behavior and satisfies the following differential equation:

$$
\frac{d y}{d t}=y(1-y)\left(\beta_{a}-\beta_{n}\right)\left(\frac{I^{A}+I^{S}}{N}\right)
$$

This can be proved using the definition of $y$ in terms of the two susceptible populations $S_{a}$ and $S_{n}$ and using the quotient rule with definitions for $\beta_{a}, \beta_{n}$ and $\beta(y)$. Note that equation (27) describes the change of behaviors distribution in susceptible individuals deriving from the different rates of infection, $\beta_{n}$ and $\beta_{a}$.

We describe this new model with the following updated system of ODEs:

$$
\begin{aligned}
\frac{d S}{d t} & =-\beta(y) S\left(\frac{I^{A}+I^{S}}{N}\right) \\
\frac{d E}{d t} & =\beta(y) S\left(\frac{I^{A}+I^{S}}{N}\right)-\sigma E \\
\frac{d I^{A}}{d t} & =(1-p) \sigma E-\omega I^{A} \\
\frac{d I^{S}}{d t} & =p \sigma E+(1-v) \omega I^{A}-\xi I^{S} \\
\frac{d H}{d t} & =q \gamma Q-\left((1-x) \alpha^{R}+x \alpha^{D}\right) H \\
\frac{d Q}{d t} & =\xi I^{S}-\gamma Q \\
\frac{d R}{d t} & =v \omega I^{A}+(1-q) \gamma Q+(1-x) \alpha^{R} H \\
\frac{d D}{d t} & =x \alpha^{D} H \\
\frac{d y}{d t} & =y(1-y)\left(\beta_{a}-\beta_{n}\right)\left(\frac{I^{A}+I^{S}}{N}\right)
\end{aligned}
$$


We now have the following theorem for the basic reproduction number for the implicit model corresponding to social behavior.

Theorem 4.1. The basic reproduction number $\mathcal{R}_{0}(y)$ for system (28)-(36) is given by

$$
\mathcal{R}_{0}(y)=\left(R_{0}^{1, n}+R_{0}^{2, n}+R_{0}^{3, n}\right) y+\left(R_{0}^{1, a}+R_{0}^{2, a}+R_{0}^{3, a}\right)(1-y)
$$

Proof. Following theorem 2.1, we can show that:

$$
R_{0}(y)=\beta(y)\left(\frac{p}{\xi}+\frac{(1-p)}{\omega}+\frac{(1-v)(1-p)}{\xi}\right)
$$

Using the definition $\beta(y)=\beta_{n}[y+r(1-y)]$ in this equation and rearranging the terms we can prove the results for $i=n, a$,

$$
\begin{aligned}
R_{0}^{1, i} & =\beta_{i} \cdot \frac{p}{\xi} \\
R_{0}^{2, i} & =\beta_{i} \cdot \frac{(1-p)}{\omega} \\
R_{0}^{3, i} & =\beta_{i} \cdot \frac{(1-v)(1-p)}{\xi}
\end{aligned}
$$

Corollary 4.2. Suppose we assume $p=1$. The basic reproduction number is given by

$$
\mathcal{R}_{0}(y)=\frac{\beta(y)}{\xi}
$$

This assumes all infectious cases are symptomatic. Hence, this basic reproduction number shows the ratio of the transmission rate as a function of human behavior to the quarantine rate.

\section{Numerical Computations}

In this section, we implement the models developed in this paper and perform numerical simulations. We implement our models in Python using the Runge-Kutta method for solving systems of ODEs. For our simulations, we implement our models for an initial population size $N(0)$ of 10,000,099, which is based on the approximate average population size of a U.S. state. We assume the number of beds $B$ is 2,700. Additionally, we assume our initial populations to be $S(0)=9,999,999, E(0)=100$, and the remaining compartments to start with zero population.

We also used the following set of parameter values for our computations from various references.

Table 2: Parameter Values

\begin{tabular}{lll}
\hline Parameter & Value & References \\
\hline$\sigma^{-1}$ & 6 days & {$[15]$} \\
$\omega^{-1}$ & 14 days & {$[15]$} \\
$\gamma^{-1}$ & 5 days & {$[5]$} \\
$\alpha^{R^{-1}}$ & 12 days & {$[2,3]$} \\
$\alpha^{D^{-1}}$ & 14 days & {$[2,3]$} \\
$q$ & 0.19 & {$[2,3]$} \\
\hline
\end{tabular}

For most of the computations, the values of the transmission probabilities were chosen to be $p=0.6$ and $v=0.8$. The value chosen for $\hat{x}=0.04$ and $r=0.8$. First, we consider the basic reproduction number that 
was derived in Theorem 2.1 and Theorem 4.1. We study the influence of the proportion of symptomatic cases, transmission rate, quarantine rate, and social behavior on the basic reproduction number.

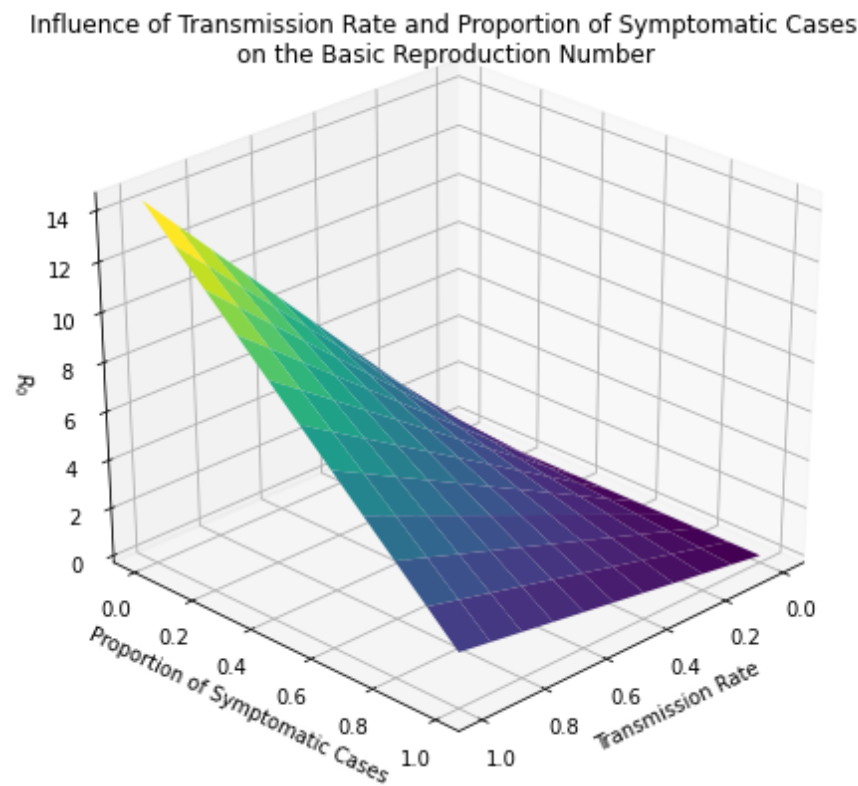

Figure 3: Influence of $p$ and $\beta$ on $R_{0}$

In Figure 3, we demonstrate the impact of the proportion of symptomatic cases, $p$, and the transmission rate, $\beta$, on $R_{0}$. Here $\xi=0.5$. Our graph indicates that a higher proportion of symptomatic cases decreases the basic reproduction number. In other words, when $p$ increase, $R_{0}^{2}$ and $R_{0}^{3}$ decreases and $R_{0}^{1}$ increases, and thus results in a lower basic reproduction number. Additionally, the graph shows there is a positive linear relationship between the transmission rate and the basic reproduction number. As the transmission rate increases, the basic reproduction number increases. Also, recall from Theorem 2.1, for the basic reproduction number to be less than 1 (which will control an epidemic [4]), we would require,

$$
\beta<\frac{1}{\frac{p}{\xi}+\frac{1-p}{\omega}+\frac{(1-v)(1-p)}{\xi}}
$$

With $\omega=1 / 14$ and $v=0.8$, this inequality suggests that a value of $\beta<0.14$ will control the COVID-19 outbreak from spreading. Figure 3 clearly illustrates this.

Furthermore, we investigate the influence of the quarantine rate on the basic reproduction number in Figure 4. Here, $\beta=0.5$ and $p=0.6$. This graph highlights the importance of a higher quarantine rate. A lower quarantine rate significantly increases the basic reproduction number.

Now, we investigate the influence of social behavior on the basic reproduction number in Figure 5 using the result from Theorem 4.1. Here $\beta_{n}=0.5, \xi=0.5$, and $p=0.6$ Again, notice a linear relationship between the proportion of normally behaved susceptibles and the basic reproduction number. As more susceptible individuals adopt normal behavior, the basic reproduction number increases. This emphasizes the importance of social distancing and confinement within the population. Thus, it is critical that the general population is encouraged to social distance to reduce the spread of COVID-19.

Figure 6 plots all eight states in system (1)-(8) and the influence of the transmission rate on the disease dynamics in the baseline model. Here, we have considered $\beta=0.5$ and $\xi=0.5$.

Figure 7 illustrates the influence of $\beta$ on the maximum number of exposed, asymptomatic, and symptomatic cases.

Next we considered the explicit model simulations and studied the influence of lockdown on disease dynamics. This is shown in Figure 8. Notice how a higher tlock increases the peak number of exposed, asymp- 


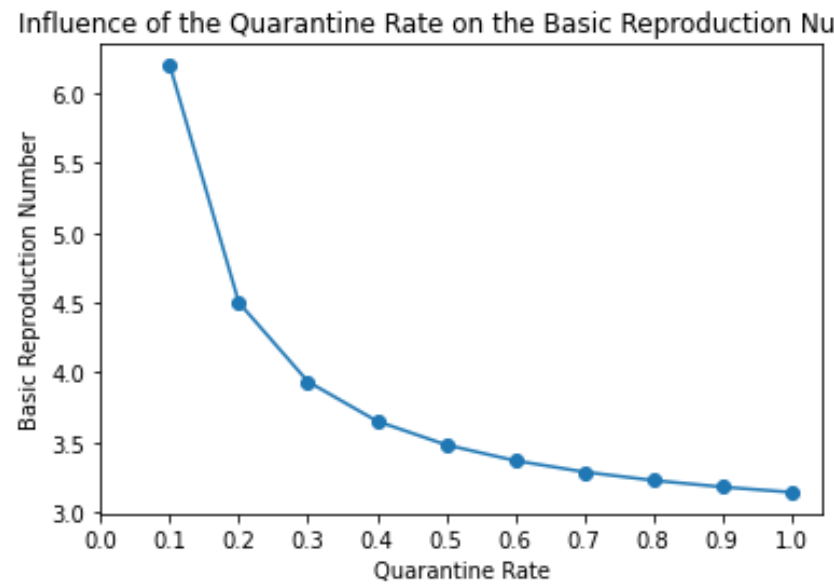

Figure 4: Influence of Quarantine Rate

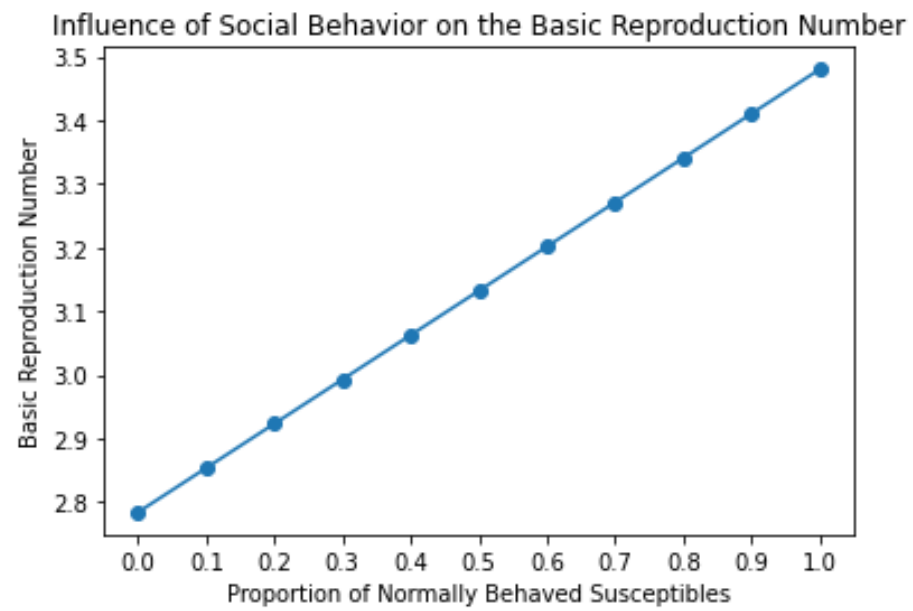

Figure 5: Influence of Social Behavior

tomatic, and symptomatic cases. Though, in our model, a tlock of 30,60, and 90 days produced similar outcomes. But, the graph indicates that tlock $=120$ days was too late, since much of the susceptible population had already been exposed and infected. This means that there were fewer confined individuals that were protected from the disease. Consequently, the number of cases was higher and peaked sooner than in the other lockdown times. Hence, sooner lockdown times protects a larger portion of the population and reduces the spread of COVID-19.

Additionally, we performed some simulations on our implicit model. The influence of the normal transmission rate on disease dynamics is shown in Figure 9. Here, we show how changing $\beta_{n}$, the normal transmission rate, impacts the peak number of exposed, asymptomatic, and symptomatic cases for the implicit model. Notably, the peaks are very similar to that of the baseline model. This can be attributed to the influence of susceptible individuals with normal behavior. Even though most susceptible individuals eventually adopt altered behavior over the course of the simulation, the effect of just susceptibles with normal behavior is similar to the effect of all susceptible individuals having normal behavior as in our baseline model.

Moreover, we observed using equation (9) when the number of hospitalizations exceeded the number of ICU beds $B=2700$. We found that by around day 65 , two months into the pandemic, the hospitalized population exceeded the number of beds for our baseline model. This corresponds to when the number of hospitalizations began to increase more quickly. When lockdown occurs after 30 days in the explicit model, it takes 68 days for the number of hospitalizations to exceed the hospital bed capacity. A much sooner lock- 

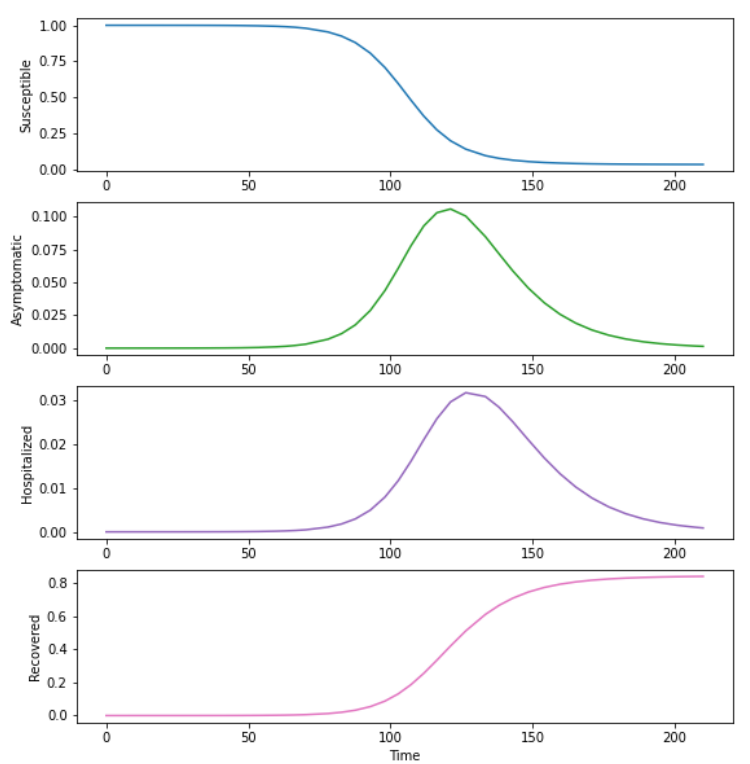
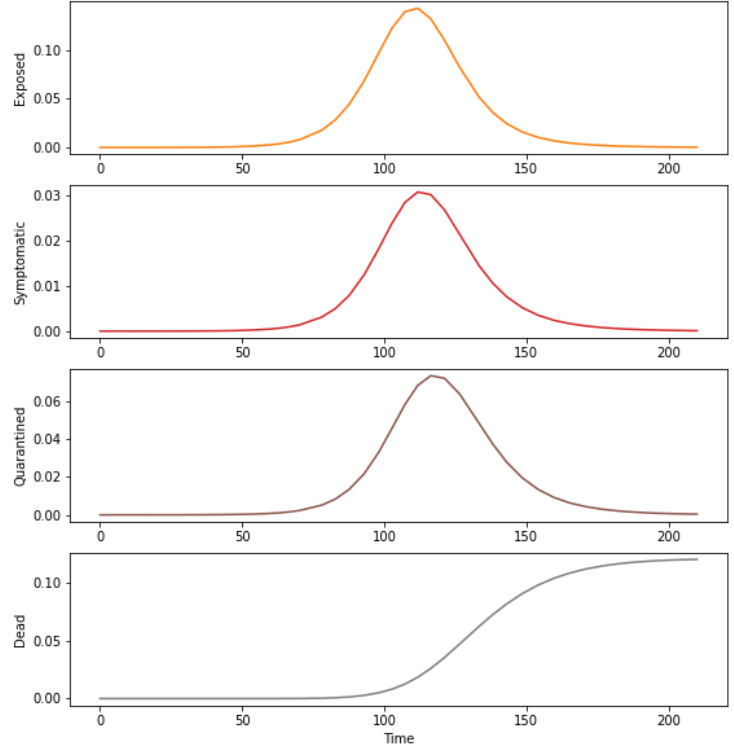

Figure 6: Dynamics of the various sub-populations in the Baseline Model including Susceptible, Exposed, Asymptomatic, Symptomatic, Hospitalized, Quarantined, Recovered and Dead

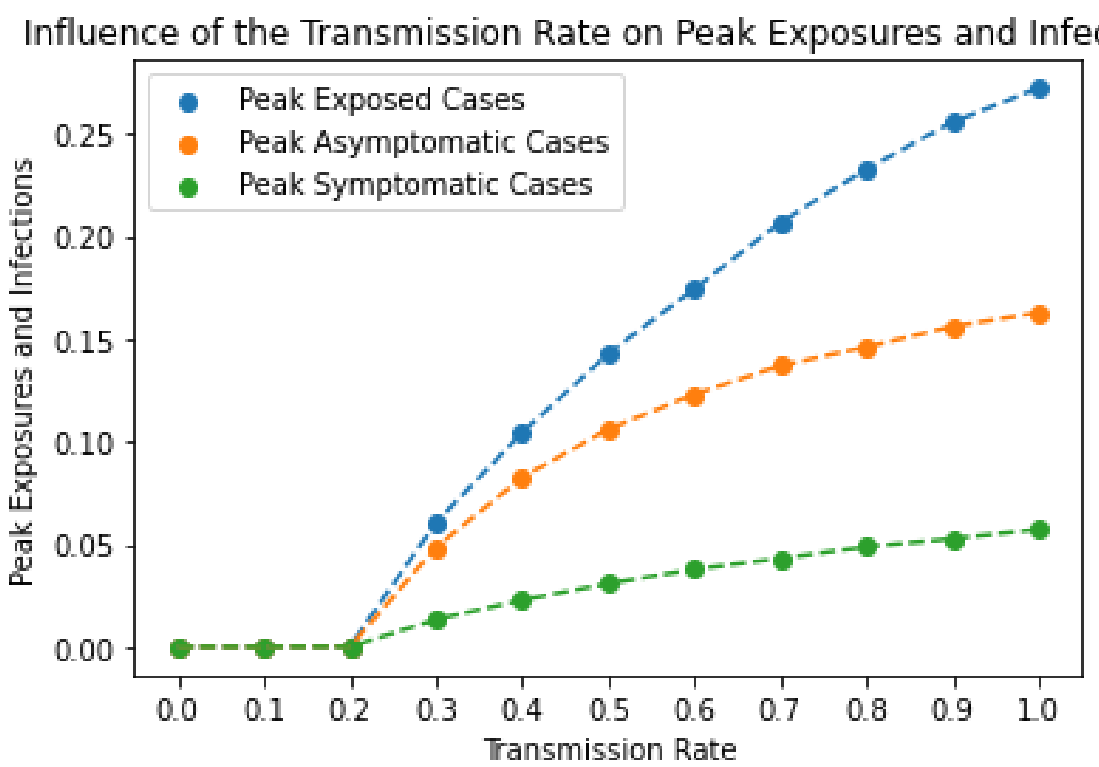

Figure 7: Baseline Model: Influence of Beta on Peak values

down, after two weeks, it takes 70 days for the number of hospitalizations to exceed the bed size. Lockdown may cause the number of hospitalizations to peak later and prolong the bed capacity.

Note that in this work, we have assumed the transmission rate $\beta$ was the same for the infected asymptomatic and infected symptomatic individuals as reflected in the first two equations of system (1)-(8). However, one could also consider the first two equations replaced as:

$$
\begin{aligned}
& \frac{d S}{d t}=-\frac{\beta_{A} S I^{A}}{N}-\frac{\beta_{S} S I^{S}}{N} \\
& \frac{d E}{d t}=\frac{\beta_{A} S I^{A}}{N}+\frac{\beta_{S} S I^{S}}{N}-\sigma E
\end{aligned}
$$



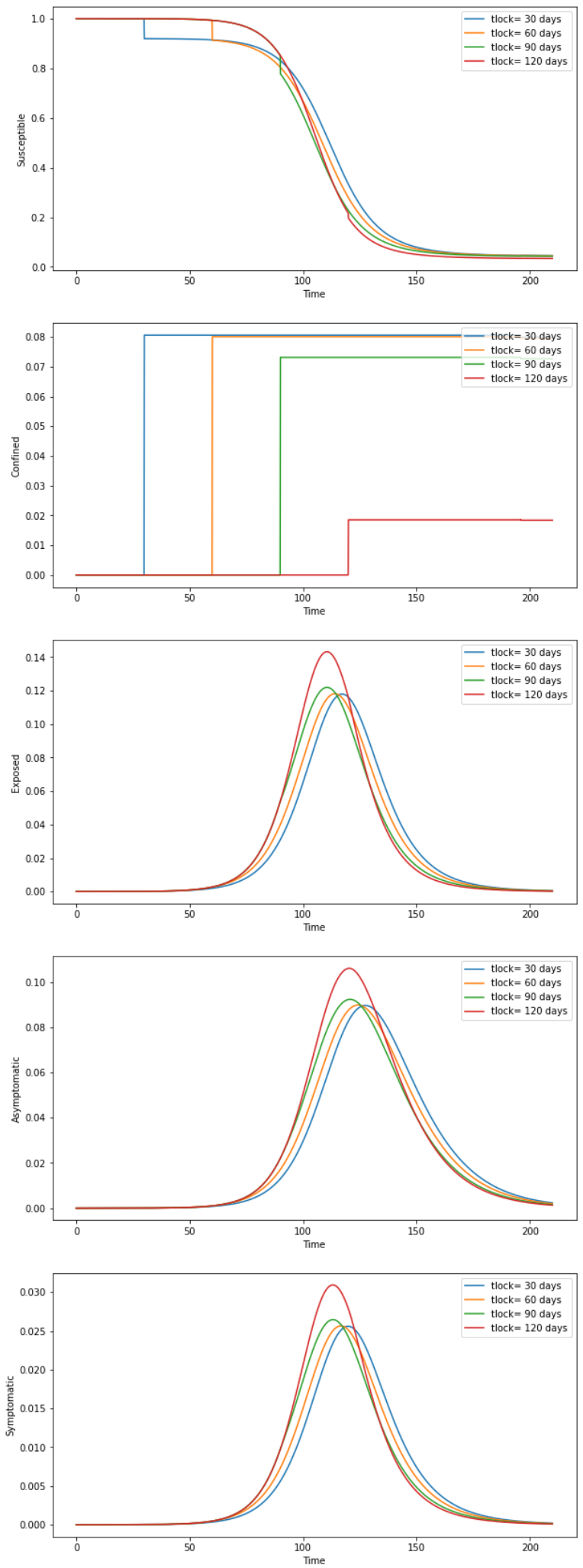

Figure 8: Explicit Model: Influence of lockdown Time on Peak values 
Influence of the Normal Transmission Rate on Peak Exposures and Infections

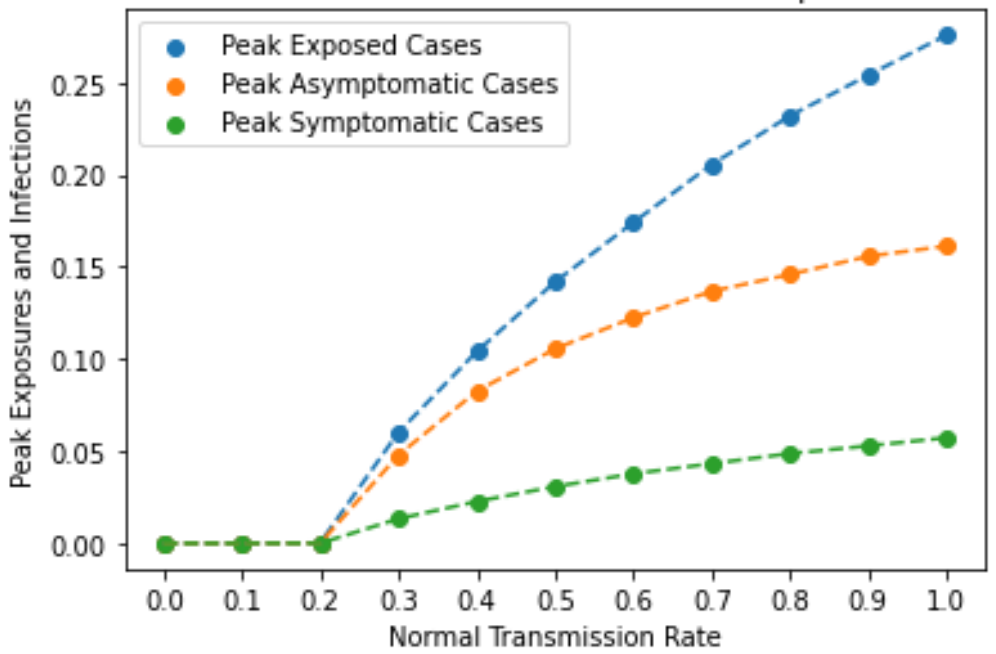

Figure 9: Implicit Model: Influence of Normal Transmission Rate on Peak values

where the transmission rate is denoted by $\beta_{A}$ for infected asymptomatic and $\beta_{S}$ for infected symptomatic individuals, respectively with $\beta_{S}=\epsilon \beta_{A}$. Here $\epsilon$ is a parameter introduced to incorporate differences in transmission rates between asymptomatic and symptomatic individuals. Figure 10 illustrates the influence of increased infectivity of asymptomatic individuals on the dynamics of the states in system (1)-(8).
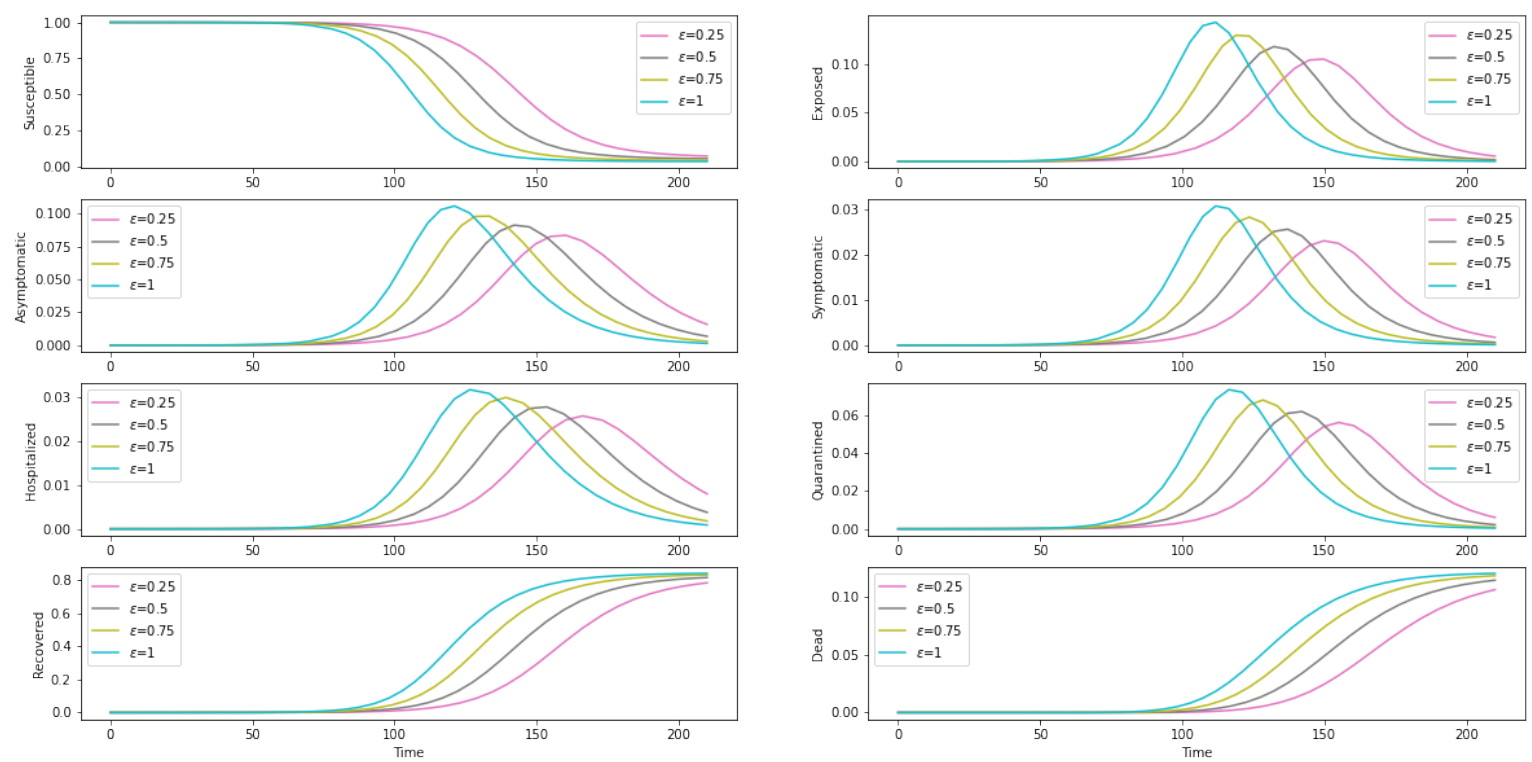

Figure 10: Baseline Model: Influence of varying transmission rates between symptomatic and asymptomatic infected populations

In addition to performing these simulations, we created graphical user interfaces for each of the models presented in this work, which are seen in Figures 11, 12, and 13. The purpose of these graphical user interfaces is for users to be able manipulate certain values and view the resulting graphs. On the left-hand side of each graphical user interface are the parameter values, which users can control. On the right-hand side, the program outputs the resulting graph. We may expand these graphical user interfaces in the future as we incor- 
porate more features into our models. Notice that such graphical user interfaces and dashboards have been extremely helpful for people across the world to make predictions and decisions regarding the pandemic.
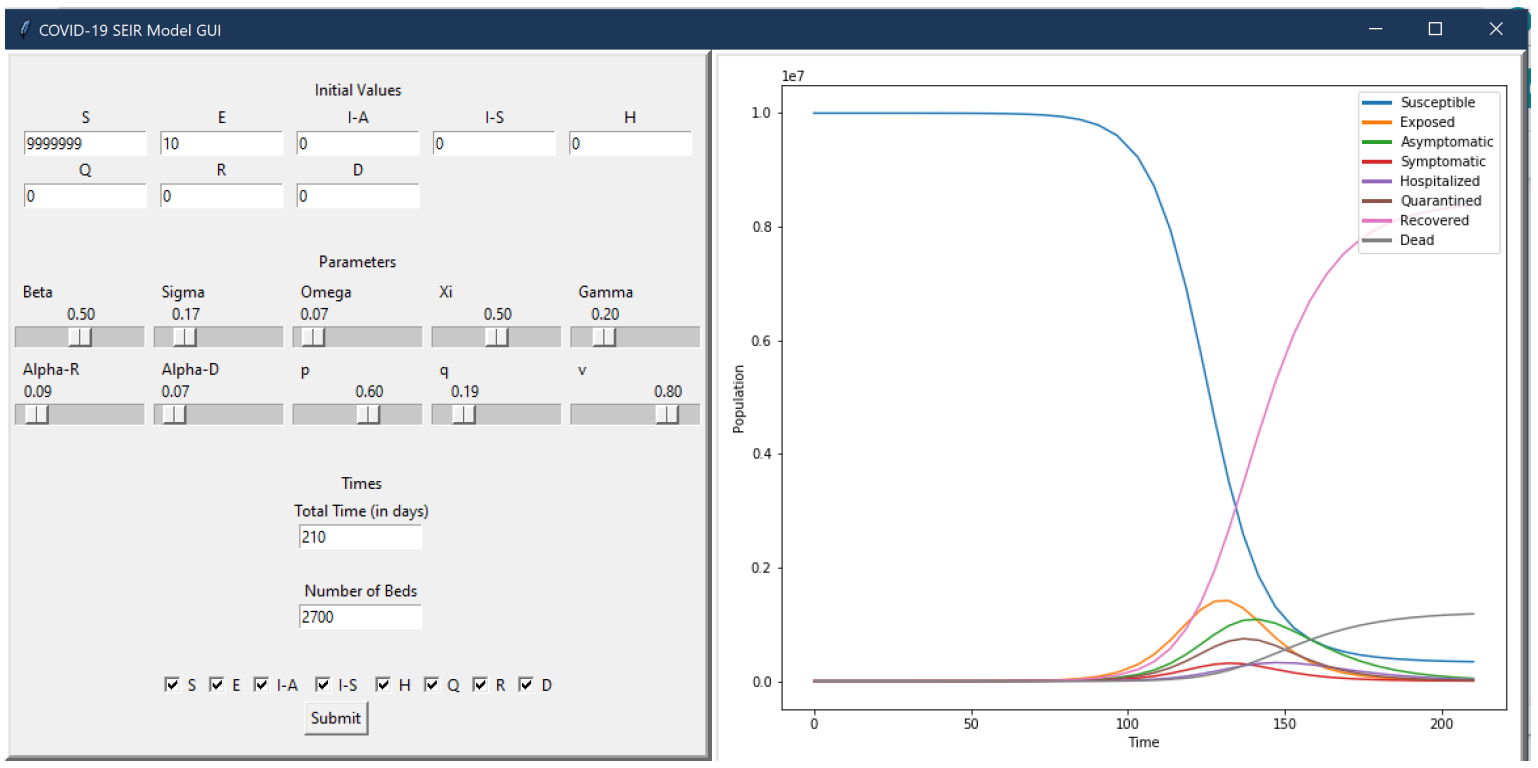

Figure 11: Baseline Model Graphical User Interface
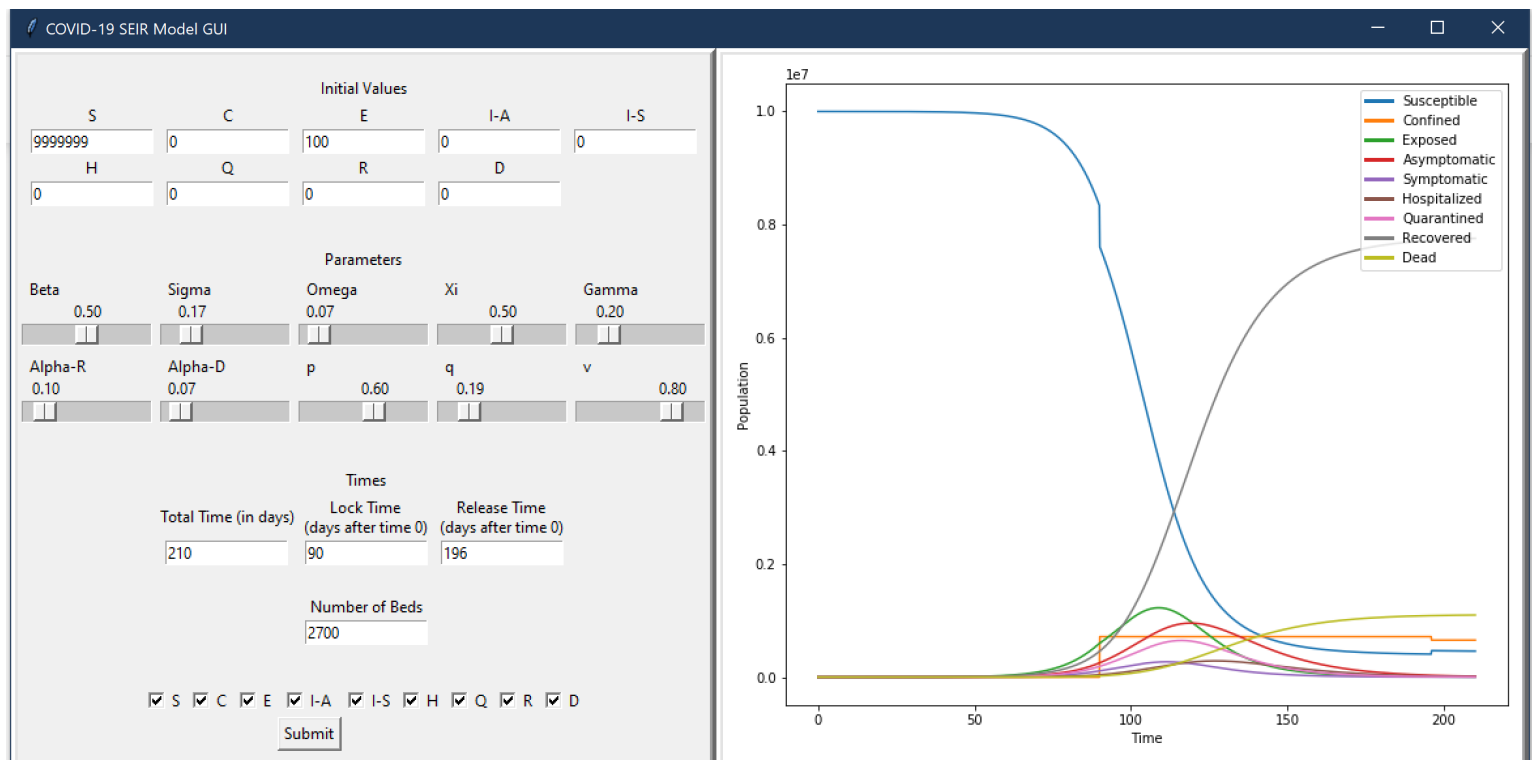

Figure 12: Explicit Model Graphical User Interface

\section{Conclusions and future work}

In this work, we considered three new epidemiological models for COVID-19 that incorporated human behaviors. Through these models, we explored adding new compartments as well as the role of both explicit and 

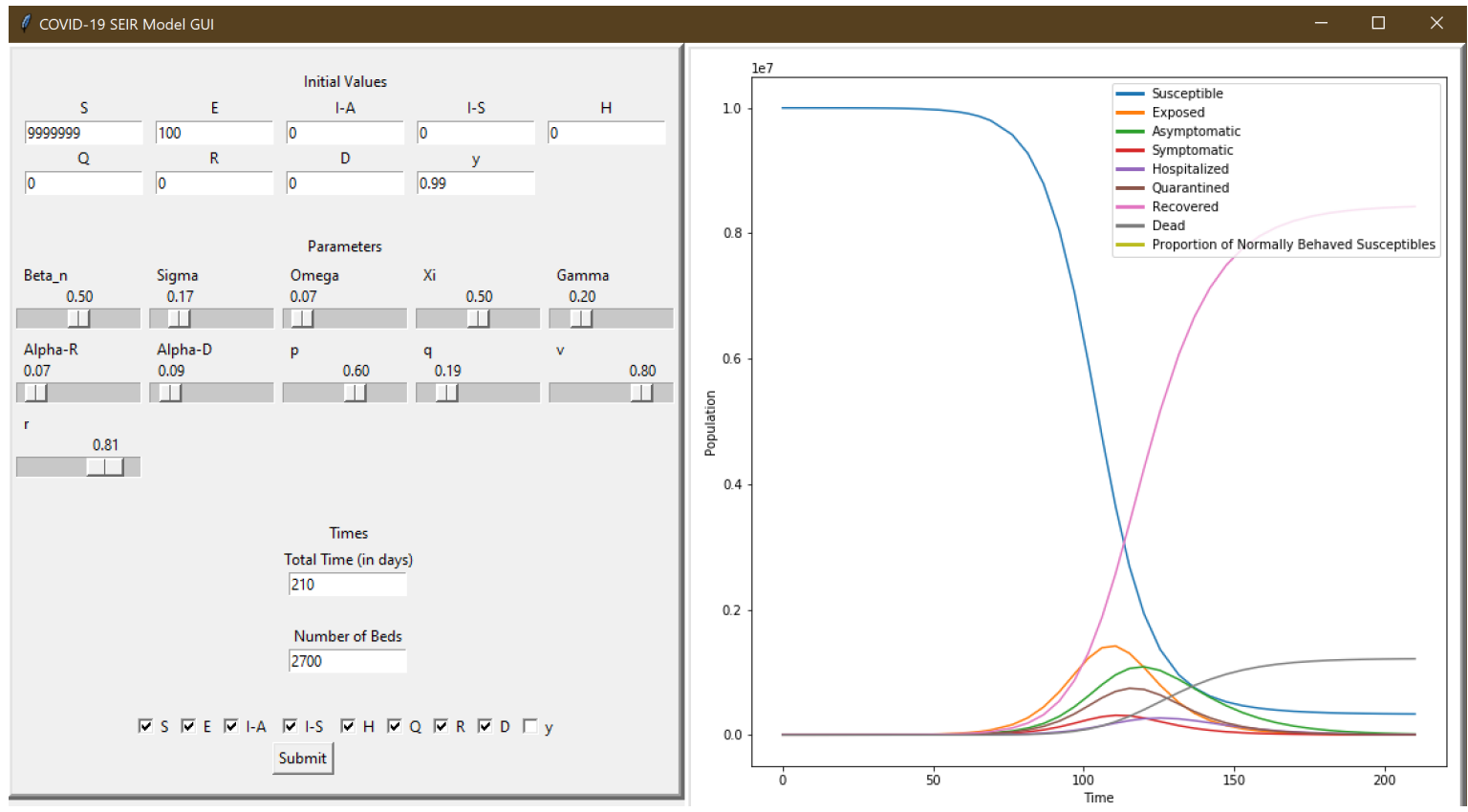

Figure 13: Implicit Model Graphical User Interface

implicit human behaviors. We also derived a basic reproduction number for our models using the Next Generation Matrix approach. Lastly, we performed numerical simulations on our models. We demonstrated how a higher proportion of symptomatic cases, lower transmission rate, lower normal transmission rate, higher quarantine rate, and lower proportion of susceptibles with normal behavior all contribute to a lower basic reproduction number. Furthermore, we showed how a lower transmission rate and sooner lockdown time reduces the peak number of cases. Our models are also able to predict ICU bed capacity requirements based on the number of days of the spread of COVID-19 which can be useful information for hospitals to plan accordingly. In addition, we explored the effects of varying the infectivity of asymptomatic and symptomatic individuals.

In the future, we plan on expanding our models. Specifically, we may add risk-perception and irrationality into our implicit model. We may also include age-structured models in behaviors and transmission rates. This will allow us to split the population into different age groups and account for different risk perceptions and other behavior related parameters. We also hope to explore the role of homogeneous and heterogeneous mixing, especially across and within age groups. Additionally, we would like to incorporate the use of face masks, which impacts the likelihood of contracting COVID-19. We also plan to investigate the level of intervention prescribed as an optimal control to manage a disease outbreak, such as when effective treatments or vaccines are not readily available or too costly to be widely used.

Acknowledgements: This work is supported in part by the National Science Foundation DMS 2031027 and DMS 2031029.

\section{Author's statement}

Conflict of interest: Authors state no conflict of interest.

\section{References}

[1] Brauer, F. \& Castillo-Chavez, C. (2001). Mathematical models in population biology and epidemiology (Vol. 40, pp. xxiv+-416). New York: Springer. 
[2] CDC. (2020a). Interim Clinical Guidance for Management of Patients with Confirmed Coronavirus Disease (COVID-19). Retrieved August 6, 2020, from Centers for Disease Control and Prevention website: https://www.cdc.gov/coronavirus/2019ncov/hcp/clinical-guidance-management-patients.html

[3] CDC. (2020b, July 10). COVID-19 Pandemic Planning Scenarios. Retrieved August 25, 2020, from Centers for Disease Control and Prevention website: https://www.cdc.gov/coronavirus/2019-ncov/hcp/planning-scenarios.html

[4] Diekmann, O., Heesterbeek, J. A. P., \& Metz, J. A. (1990). On the definition and the computation of the basic reproduction ratio $\mathrm{R} 0$ in models for infectious diseases in heterogeneous populations. Journal of mathematical biology, 28(4), 365-382.

[5] Ferguson, N.M., Laydon, D., Nedjati-Gilani, G., Imai, N., Ainslie, K., Baguelin, M., Bhatia, S., Boonyasiri, A., Cucunubá, Z., Cuomo-Dannenburg, G. and Dighe, A., (2020). Impact of non-pharmaceutical interventions (NPIs) to reduce COVID-19 mortality and healthcare demand. 5. https://doi.org/10.25561/77482.

[6] Kucharski, A.J., Russell, T.W., Diamond, C., Liu, Y., Edmunds, J., Funk, S., Eggo, R.M., Sun, F., Jit, M., Munday, J.D. \& Davies, N., (2020). Early dynamics of transmission and control of COVID-19: a mathematical modelling study. The lancet infectious diseases.

[7] Kim, Y., Lee, S., Chu, C., Choe, S., Hong, S., \& Shin, Y. (2016). The characteristics of Middle Eastern respiratory syndrome coronavirus transmission dynamics in South Korea. Osong public health and research perspectives, 7(1), 49-55.

[8] Li, R., Pei, S., Chen, B., Song, Y., Zhang, T., Yang, W., \& Shaman, J. (2020). Substantial undocumented infection facilitates the rapid dissemination of novel coronavirus (SARS-CoV-2). Science, 368(6490), 489-493.

[9] Liu, Z., Magal, P., Seydi, O., \& Webb, G. (2020). A COVID-19 epidemic model with latency period. Infectious Disease Modelling, 5, 323-337.

[10] Maier, B. F., \& Brockmann, D. (2020). Effective containment explains subexponential growth in recent confirmed COVID-19 cases in China. Science, 368(6492), 742-746.

[11] K. Prem, Y. Y. Liu, T. Russell, A. Kucharski, R. Eggo, N. Davies, S. Flasche, S. Clifford, C. Pearson, J. Munday, S. Abbott, H. Gibbs, A. Rosello, B. Quilty, T. Jombart, F. Sun, C. Diamond, A. Gimma, K. van Zandvoort, S. Funk, C. Jarvis, J. Edmunds, N. Bosse, J. Hellewell, M. Jit, and P. Klepac (2020) "The effect of control strategies to reduce social mixing on outcomes of the COVID-19 epidemic in Wuhan, China: a modelling study." In: The Lancet Public Health, doi: 10.1016/S2468-2667(20)30073-6.

[12] Sohrabi, C., Alsafi, Z., O’Neill, N., Khan, M., Kerwan, A., Al-Jabir, A., losifidis, C. \& Agha, R. (2020). World Health Organization declares global emergency: A review of the 2019 novel coronavirus (COVID-19). International Journal of Surgery.

[13] Roosa, K., Lee, Y., Luo, R., Kirpich, A., Rothenberg, R., Hyman, J. M., Yan, P. \& Chowell, G. (2020). Real-time forecasts of the COVID-19 epidemic in China from February 5th to February 24th, 2020. Infectious Disease Modelling, 5, 256-263.

[14] Wang, C., Horby, P. W., Hayden, F. G., \& Gao, G. F. (2020). A novel coronavirus outbreak of global health concern. The Lancet, 395(10223), 470-473.

[15] WHO. (2020). Report of the WHO-China Joint Mission on Coronavirus Disease 2019 (COVID-19). In World Health Organization (p. 12). Retrieved from https://www.who.int/docs/default-source/coronaviruse/who-china-joint-mission-on-covid-19-finalreport.pdf 